\title{
78. ADDITIONAL EVIDENCE FOR THE CATACLYSMIC ORIGIN OF SPIRAL STRUCTURE IN GALAXIES
}

\author{
K. R U DNICK I \\ Warsaw University Observatory, Warsaw, Poland
}

\begin{abstract}
The photograph of the galaxy NGC 3486 shows its spiral structure in statu nascendi. The evident explanation is that of a cataclysmic origin of the visible spiral arms.

The photograph of the galaxy NGC 3486 (right ascension $=10^{\mathrm{h}} 57^{\mathrm{m}} \cdot 6$, declination $=+$ $29^{\circ} 15$ according to Zwicky and Herzog (1963) taken with the $125 \mathrm{~cm}$ Palomar Schmidt Telescope is presented (Figure 1). Spiral arms of this galaxy have plenty of splits which form a secondary spiral structure. Split spiral arms in galaxies are well known. Many of them are shown in Arp's Atlas 1966 (e.g. Nos: 7,9, 25, 26, 29, 41,108,113,114). In NGC 3486, however, the secondary arms form in many cases almost right angles with the primary arms at the connection points. Thus any explanation of the origin of such spiral structure by a gravitational instability or by an electromagnetic field is rather unlikely. The secondary arms seem to be jets curved subsequently due to the rotation. Since no basic difference is visible in appearance of both the primary and the secondary spiral arms, this can serve as an additional argument in favour of a cataclysmic origin of the spiral structure of galaxies in general.
\end{abstract}

Fig. 1. A photograph of NGC 3486 obtained with the $125 \mathrm{~cm}$ Palomar Schmidt telescope. Emulsion Kodak 103a-0, without filter. Date 1962, April 1, $6^{\mathrm{h}} 25^{\mathrm{m}}$ UT; exposure time $9 \mathrm{~min}$. North is at the top east to the left. Scale: $6.5 \mathrm{~mm}=1 \mathrm{~min}$ of arc.

Even if we interpret the complicated structure of NGC 3486 as a superposition of two spiral structures of independent origins, the cataclysmic explanation of them seems again the most probable one. 


\section{References}

Arp, H.: 1966, Astrophys. J. Suppl. Ser. 14, 1.

Zwicky, F. and Herzog, E.: 1963, Catalogue of Galaxies and Clusters of Galaxies, Vol. II, California Institute of Technology. 